\title{
TRANSLATION OF SYNTAX OF OPERATION MANUAL FROM ENGLISH INTO RUSSIAN
}

\author{
Staroverova A. D. (Russian Federation)
}

\author{
Staroverova Anastasia Dmitrievna - student of Master's programme, \\ department of translation and intercultural communication, faculty of foreign languages, \\ Tula state pedagogical University named after Leo Tolstoy, Tula
}

\begin{abstract}
English into the Russian language. It is found out how sentences are changed from the point of view of their structure and components. Possible syntax changes are classified and analyzed from the point of view of their appropriateness according to the idiomatic nature and all-Union State Standard. The practical value of the article is to help young professionals to avoid calquing while translating manuals.
\end{abstract}

Keywords: manual, syntax, translation, operation.

\section{ПЕРЕВОД СИНТАКСИСА ТЕКСТОВ ИНСТРУКЦИЙ ПО ЭКСПЛУАТАЦИИ С АНГЛИЙСКОГО ЯЗЫКА НА РУССКИЙ Староверова А. Д. (Российская Федерация)}

\author{
Староверова Анастасия Дмитриевна - студент магистратуры, \\ кафедра переводоведения и межкультурной коммуникаичи, факультет иностранных языков, \\ Тульский государственный педагогический университет им. Л. Н.Толстого, г. Тула
}

\begin{abstract}
Аннотация: в данной статье анализируются способы перевода синтаксических конструкций инструкции по эксплуатации шлифовального прибора с английского языка на русский, выявляется, как предложения меняются по составу и как меняется синтаксическая роль членов предложения. Приводится классификация возможных способов синтаксических замен и анализируется правомерность проведения тех или иных трансформаџий с точки зрения требований ГОСТа и идиоматичности. Практическая значимость данной статьи состоит в том, чтобы помочь молодым переводчикам избежать калькирования при переводе инструкиий.
\end{abstract}

Ключевые слова: инструкция, синтаксис, перевод, эксплуатация.

Научно-технический прогресс шагнул далеко. Каждый день появляются новые приборы и инструменты, которые, благодаря глобализации, получают распространение не только в странепроизводителе, но и во всем мире. В связи с этим возрастает роль грамотного перевода всевозможных документов, сопровождающих новое устройство.

В данном исследовании мы обратимся к анализу перевода синтаксиса текстов инструкций приборов, применяемых в строительстве.

Цель данного исследования - выявить, как предложения меняются по составу и как меняются роли членов предложения при переводе.

Практическая значимость данной статьи состоит в том, чтобы помочь будущим студентампереводчикам избежать калькирования и делать текст более идиоматичным. Не бояться изменять порядок слов или синтаксическую функцию предложения, не смотря на требование точности, характерное для текста инструкций. Также мы рассмотрим не самые удачные, на наш взгляд, варианты перевода и изложим свое видение.

В данной статье будут изложены результаты анализа инструкции угловой шлифовальной машины G 10SR3, G 12SR3, G 13SR3 компании Hitachi [3].

Инструкция не очень объемная, мы проанализировали всю и здесь остановимся на некоторых моментах перевода синтаксиса, представляющих для нас наибольший интерес, то есть тех предложениях, где производились какие-либо замены. Подчеркнем, что мы будем говорить только о синтаксических изменениях, мы не затронем морфологию и лексику.

Из курса языкознания нам известно, что синтаксические структуры русского и английского языка различаются, что и приводит к определенным заменам при переводе синтаксиса [1].

1) Изменение роли членов предложения, например:

дополнение $\rightarrow$ подлежащее

The following show symbols used for the machine (c. 3).

Ниже приведены символь, используемые для машины (с. 5);

- обстоятельство места $\rightarrow$ обстоятельство образа действия

... must be collected separately and returned to an environmentally compatible recycling facilities (c. 3 ).

... должны утилизовываться отдельно безопасным для окружающей среды образом (с. 5); 
- дополнение $\rightarrow$ обстоятельство места

Keep your work area clean and well lit (c. 6, 1a).

Поддерживайте чистоту и хорошее освещение на рабочем месте (c. 76, 1a);

- дополнение $\rightarrow$ сказуемое

Use extra caution when making a "pocket cut" into existing walls or other blind areas (c. 8, f).

Будьте предельно осторожны, проделывая «карманный надрез» в существующей стене или отмостке (c. 79, f).

2) Предложения могут изменить отражение отношений между явлениями реальной действительности, то есть превратиться из утвердительного в отрицательное:

Avoid body contact with earthed or grounded surfaces, such as pipes, radiators, ranges and refrigerators (c. 6 , 2b).

Не прикасайтесь телом к заземленным поверхностям, например, к трубопроводам радиаторам, кухонным плитам и холодильникам (c.76, 2b).

Или, наоборот - из отрицательного в утвердительное:

Do not abuse the cord (c. 6, 2d).

Правильно обращайтесь со шнуром (с. 76, 2d).

3) Изменение порядка

- частей предложения:

There is an increased risk of electric shock if your body is earthed or grounded (c. 6, 2b).

Если ваше тело соприкоснется с заземленными поверхностями, возрастет опасность поражения электрическим током (c. 76, 2b).

Confirm that the push button is disengaged by pushing the push button two or three times before switching the power tool on (c. 9, point 7).

Перед включением электрического инструмента проверьте нажимную кнопку и убедитесь в том, что нажимная кнопка освобождена, путем двух- или трехразового нажатия (с. 81, пункт 7).

Should any of the screws be loose, retighten them immediately (c. 10, Maintenance and inspection, point 2).

Немедленно затяните винт, который окажется ослабленным (с.82, пункт 2).

- однородных членов предложения:

Ensure the switch is in the off-position before connecting to power source and/or battery pack, picking up or carrying the tool (c. 6, 3c).

Убедитесь в том, что выключатель находится в положения выключения перед подниманием, переноской или подсоединением к сетевой розетке и/или портативному батарейному источнику питания (c. 76, 3c).

Прокомментируем последние два способа. На наш взгляд, изменение порядка однородных членов в данном случае не вполне обосновано, хотя никак не нарушает логичность построения. Когда мы говорим об адекватном переводе, мы говорим и об уровнях эквивалентности, и если есть возможность сохранить заданную оригиналом структуру, мы должны это делать [4]. Например, в следующем примере:

Check that speed marked on the wheel is equal to or greater than the rated speed of the grinder (c. 8, "General safety instructions").

Убедитесь, что скорость, указанная на шлифовальном круге, больше или равна номинальной скорости шлифовальной машины (с. 79, «Общие правила безопасности»).

Сохранить порядок было невозможно, потому что перевод станет неидиоматичным. В русском языке есть два устойчивых выражения «Больше или равен» и «меньше или равен».

Замена порядка частей сложного предложения была произведена верно: в переводе мы не должны забывать о тема-рематическом членении предложения. В английском языке на первом месте новая информация (рема), на втором - старая (тема). В русском - наоборот.

4) Изменение залога и времени

Для английского языка характерно более частое употребление пассивного залога, чем в русском языке.

Any power tool that cannot be controlled with the switch is dangerous and must be repaired (c. 6, 4b).

Каждый электроинструмент, которым нельзя управлять с помощью выключателя, будет представлять опасность, и его будут необходимо отремонтировать.

Пассивный налог настоящего времени $\rightarrow$ активный залог будущего времени (c. 77, 4b).

Говоря о времени глагола, следует упомянуть, что в тексте инструкций всегда используется настоящее время глагола, чтобы подчеркнуть общность и стандартность явления, поэтому в следующем примере переводчик поступил абсолютно верно [5]:

Damaged accessories will normally break apart during this test time (c. 7, g).

Во время такой проверки, поврежденные принадлежности обычно разбиваются (с. 78, g).

Будущее время $\rightarrow$ возвратная конструкция в настоящем времени.

5) Изменение структуры предложения 
Одним из главных критериев любой инструкции является ее четкость и понятность, поэтому для выполнения этого условия переводчик может разбивать или объединять предложения [2]. Например,

- простое предложение с деепричастным оборотом $\rightarrow$ сложноподчиненное

The eyes protection must be capable of stopping flying flying debris generated by various operations. The dust mask or respirator must be capable of filtrating particles generated by your operations (c. $7, \mathrm{~h}$ ).

Средства защиты глаз должны защищать глаза от попадания летящих обрезков, которые возникают при выполнении различных видов работ. Лицевая маска или респиратор должны отфильтровывать частицы пыли, которые образуются во время работы (с. 78, h).

- замена простого предложения $\rightarrow$ сложноподчиненное

The guard must be securely attached to the power tool and positioned for maximum safety, so the least amount of wheel is exposed towards the operator (c. 8, "Safety warnings", b).

Защитное приспособление должно быть надежно прикреплено $\kappa$ электроинструменту $u$ установлено таким образом, [зачем?] чтобы достичь максимальной безопасности, с тем, чтобы наименьшая часть круга была обращена к оператору (с. 79, «Особые меры предосторожности», b). одно предложение $\rightarrow$ два предложения

When the work area is removed from the power source, use an extension cord of sufficient thickness and rated capacity (c. 9, point 3 ).

Когда рабочая площадка удалена от источника электропитания, пользуйтесь удлинителем. Удлинитель должен иметь требуемую площадь поперечного сечения и обеспечивать работу инструмента заданной мощности (с. 80, пункт 3).

- два предложения $\rightarrow$ одно

Hitachi Power Tools are constantly being improved and modified to incorporate the latest technological advancements. Accordingly, some parts (i.e. code numbers and/or design) may be changed without prior notice. (c. 11, Modifications)

Фирма Hitachi непрерывно работает над усовершенствованием своих изделий, поэтому мы сохраняем за собой право на внесение изменений в технические характеристики, упомянутые в данной инструкции по эксплуатации, без предупреждения об этом (с. 82, Замечание).

Таким образом, мы выделили 6 основных синтаксических трансформаций при переводе инструкций техники по эксплуатации прибора: изменение роли членов предложения, изменение порядка, изменение залога, изменение времени, изменение структуры предложения

При написании данной статьи мы выписали все примеры синтаксических трансформаций (в тексте инструкции было еще 5 примеров, которые мы не стали выписывать в силу схожести с приведенными выше). Как видно, их не так уж и много. То есть, большая часть трансформаций приходится на уровень морфологии и лексики. Но, тем не менее, переводчик должен обращать внимание на синтаксис предложения, потому ни один идеально подобранный эквивалент не поможет читателю быстро и точно понять предложение, если его структура плохо организована или предложение вообще нечитаемо.

\section{Список литературы / References}

1. Бархударов Л. С. Язык и перевод (Вопросы общей и частной теории перевода). М., Междунар. отношения, 1975. $240 \mathrm{c}$.

2. ГОСТ 2.105-95 Единая система конструкторской документации (ЕСКД). Общие требования к текстовым документам (с Изменением № 1, с Поправками). Введ. 1996-01-01. М.: Изд-во стандартов, 1996. 29 с.

3. Инструкция угловой шлифовальной машины G 10SR3, G 12SR3, G 13SR3 компании Hitachi. Printed in China, 2013. $90 \mathrm{c}$.

4. Комиссаров В. Н. Современное переводоведение. М.:ЭТС, 2002. 424 с.

5. Максимов В. И. Русский язык и культура речи: Учебник. М.: Гардарики, 2001. 413 с.

\section{Список литературы на английском языке / References in English}

1. Barhudarov L. S. Jazyk i perevod [Language and translation] (Voprosy obshhej i chastnoj teorii perevoda) [Issues of general and subtheory of translation]. - M., Mezhdunar. otnoshenija, 1975. 240 p. [in Russian]

2. GOST 2.105-95 Edinaja sistema konstruktorskoj dokumentacii (ESKD). Obshhie trebovanija k tekstovym dokumentam (s Izmeneniem N 1, s Popravkami) [The United system of construction documents (USCD). General requirements for text documents (with change N 1 and amendmants)] Vved. 1996-01-01. M.: Izd-vo standartov, 1996. 29 p.

3. Instrukcija uglovoj shlifoval'noj mashiny G 10SR3, G 12SR3, G 13SR3 kompanii Hitachi [Operation manual for disc grinder G 10SR3, G 12SR3, G 13SR3 of Hitachi company]. Printed in China, 2013. 90 p.

4. Komissarov V. N. Sovremennoe perevodovedenie [Modern theory of translation]. M.:JeTS, 2002. 424 p. 
5. Maksimov V. I. Russkij jazyk i kul'tura rechi: Uchebnik [The Russian language and culture of speech: Student's book]. M.: Gardariki, 2001. 413 p. 